\title{
A dimensão institucional do processo de crescimento econômico: inovações e mudanças institucionais, rotinas e tecnologia social ${ }^{1}$
}

\author{
Octavio A. C. Conceição ${ }^{2}$
}

\section{Resumo}

Este artigo trata da natureza do processo de crescimento econômico, como resultado da interação entre a mudança institucional e tecnológica, o papel das firmas e as instituições. Tal perspectiva analítica se faz presente tanto nas abordagens evolucionárias, quanto institucionalistas. Elas comungam em dois aspectos: a) desenvolvimento é um processo multifacetado, que exige a investigação das condições que permitem seu avanço de maneira historicamente diferenciada; e b) a mudança tecnológica, as características das firmas e as instituições, em conjunto, moldam padrões específicos de desenvolvimento. Nesse sentido discutiremos a contribuição de Douglass North, Matthews, Zysman e Nelson.

Palavras-chave: Economia institucional; Economia evolucionária; Desenvolvimento econômico.

\begin{abstract}
The institutional dimension of the economic growth process: innovation and institutional change, routines and social

This paper deals with the nature of the economic growth process, given by the interaction of institutional and technological change, the role of firms and institutions. This analytical perspective is present in both the evolutionary and the institutionalist approach. They both agree in two points of view: a) development is a complex process which claims for an inquiry about the conditions allowing for advancing through historically differentiated avenues; and b) technological change, firms and institutions jointly define specific development patterns. From this perspective the contributions of Douglass North, Matthews, Zysman and Nelson are discussed.
\end{abstract}

Key words: Institutional economics; Evolutionary economics; Economic development.

JEL B25, E11.

To build new theories one must necessarily be critical of older theories. Otherwise the old theory will not give space for new. Unless the new theory can make claims of comparative superiority then it will not be graced with a readership. Science often develops as the result of dispute and debate. In this manner even wrong theories can be of positive value: they help us clarify what is required to surpass their defects (Hodgson, 2001, p. 270).

\section{Introdução}

O conceito de instituição, enquanto conjunto de normas, regras, hábitos e sua evolução (Hodgson, 1998; North, 1990; e Nelson, 1995), vem assumindo uma

(1) Trabalho recebido em dezembro de 2006 e aprovado em setembro de 2007.

(2) Economista da Fundação de Economia e Estatística (FEE); Professor adjunto do Programa de PósGraduação em Economia da Universidade Federal do Rio Grande do Sul. E-mail: < octavio@ fee.tche.br>. 
significativa, inovadora e desafiadora dimensão analítica, quando inserido em diferentes contextos. A partir daí se constata que a própria noção de instituição, em função das raízes históricas e estruturais que lhe são específicas, passa a viabilizar distintas trajetórias de crescimento, em seus respectivos ambientes socioeconômicos. Por esta razão instituição e crescimento econômico são conceitos umbilicalmente vinculados. Entretanto, qual a relação entre ambos? A resposta a essa questão envolve uma discussão a respeito do conceito, da forma, e do tratamento teórico dispensado às instituições, que assume diferentes conotações, conforme o modelo de crescimento econômico adotado. São destas diferenças que buscaremos tratar nesse artigo. Para tanto, o subdividiremos em 4 partes: na primeira proceder-se-á a uma breve discussão da importância que os institucionalistas conferem à complexa questão do processo de crescimento econômico.

Na parte 2 do artigo será analisada a "evolução" do marco teórico institucionalista, a partir da contribuição de três autores selecionados, que explicitam pontos relevantes da referida evolução teórica. A linha analítica aí desenvolvida procurará demonstrar a forma através da qual as modernas vertentes do institucionalismo trataram as questões relativas à dimensão do processo de crescimento econômico. Tal perspectiva, como será examinado, sugere uma nítida confluência com o evolucionismo. Vale dizer, as modernas vertentes institucionalistas (NEI e Neo-institucionalistas) vêm revelando uma clara convergência teórica com a agenda de pesquisa evolucionária sobre crescimento econômico, conforme proposição feita por Richard Nelson (1998). Isto também sintoniza com os princípios evolucionários implícitos ao pensamento de Veblen, os quais constituem a principal referência da corrente aqui denominada de Neoinstitucionalismo. Senão vejamos.

Matthews, em seu trabalho seminal de 1986, tem uma filiação muito próxima à Nova Economia Institucional (NEI), e estabeleceu princípios importantes à compreensão das mudanças institucionais, distinguindo-as fundamentalmente de qualquer convergência a situações de ótimo paretiano. Tal assertiva qualificou o debate e permitiu novos avanços nesse sentido, embora se reconheça que a referida abordagem não tenha a menor intenção de romper com o pensamento neoclássico. Zysman (1994), sob uma perspectiva analítica Neoinstitucionalista - a qual, segundo Samuels (1995), poderia, sem grandes transtornos metodológicos, ser também designada de "institucionalismo evolucionário" - fortalece os laços de uma absoluta compatibilidade (e identidade) entre institucionalismo e evolucionismo, desde que resguardada a necessidade de ruptura com o paradigma neoclássico. Douglass North (1990 e 2005), um dos mais proeminentes autores da NEI, vem reforçando cada vez mais claramente uma crescente vinculação de sua corrente com o evolucionismo. A análise da 
contribuição desses três autores e em três momentos históricos distintos - a saber, Matthews (1986), Zysman (1994) e North (1990 e 2005) - terá, por objetivo, estabelecer pontos relevantes, os quais passaram a ser norteadores da evolução teórica do respectivo campo de pesquisa.

Na parte 3 do artigo buscar-se-á discutir a forma como a abordagem evolucionária (ou neo-schumpeteriana) incorpora as referidas noções. Essa discussão não tem por meta, naturalmente, esgotar todo o rico instrumental analítico desenvolvido, ao longo das últimas duas décadas e meia, pela bemsucedida tradição neo-schumpeteriana ou evolucionária. Mas visa, isso sim, a tentar sistematizar, a partir da contribuição de Nelson (2002), os pontos considerados essenciais à constituição de uma nova "teoria de crescimento econômico", com a inclusão das instituições no referido processo. ${ }^{3}$ Sua abordagem permite integrar aspectos tanto micro, quanto macroeconômicos, vinculando, de maneira mais estrita, o processo de crescimento ao ambiente tecnológico, às mudanças estruturais, à inovatividade e, naturalmente, ao desenho institucional que lhe sustenta.

No último item, incluem-se algumas considerações finais. Mais uma vez é importante salientar que tal discussão buscará demonstrar que a agenda de pesquisa institucionalista sobre crescimento econômico tem, em muitos aspectos, notável convergência com o evolucionismo. Embora ambas representem abordagens distintas, elas não são incompatíveis.

\section{0 crescimento nos institucionalistas}

As ponderações acima enfatizam questões que, a nosso ver, nunca deveriam ter sido formalmente omitidas dentro do moderno pensamento econômico. Dentre essas se destacam as que contemplam: (a) crescimento econômico como um processo, que, enquanto tal, pressupõe rupturas e reconstruções; (b) as características da transição de um velho para um novo processo de crescimento como elementos decisivos para a análise; (c) a importância das mudanças estruturais de natureza tanto tecnológica, quanto institucional; e (d) a visão do referido processo como revestido, de país para país, e de região para região, de características bastante distintas e, às vezes, sequer

(3) Não se quer, com isso, excluir os demais autores neo-schumpeterianos do referido debate, já que as contribuições de autores como Giovanni Dosi, Jan Fagerberg, Christopher Freeman, Luigi Orsenigo, dentre outros, ajudaram a constituir o precioso legado dessa abordagem. A escolha de Richard Nelson como principal referência evolucionista neste artigo deve-se ao fato de o referido autor, além de ícone da contribuição neoschumpeteriana, em parceria com Sidney Winter, vir, nos últimos anos, trabalhando ativamente na constituição de uma agenda de pesquisa sobre a relação entre instituições e crescimento econômico. Vide, em especial, seu artigo (Nelson, 1998) publicado no Cambridge Journal of Economics, em edição organizada por Geoffrey Hodgson, quando da comemoração do centenário de publicação do clássico de Thorstein Veblen "Why is economics not an evolutionary science". 


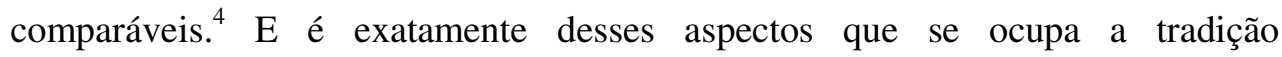
institucionalista: a história importa, as formas de crescimento capitalistas são diferenciadas e múltiplas, o processo de crescimento é contínuo e tem raízes históricas profundas (North, 2005; Hodgson, 2002).

Para incorporar teoricamente a noção de crescimento econômico nos institucionalistas algumas considerações são decisivas. Em que pese o grande número de estudos recentes que explicitam os elementos centrais de uma análise institucionalista, pode-se, genericamente, agrupá-las em três approaches: o Antigo Institucionalismo Norte-Americano de Veblen, Commons e Mitchell; a Nova Economia Institucional de Coase, Williamson e North; e o Neo-Institucionalismo de Hodgson, Samuels e Rutherford (Samuels, 1995, Hodgson, 1993). ${ }^{5}$ Há nessas três abordagens alguns pontos consensuais no que tange ao papel das instituições no referido processo, mas também grandes divergências, principalmente na comparação dos Antigos e Neo-institucionalistas com os economistas da NEI.

Como já salientado, a semelhança entre essas três correntes se dá, em primeiro lugar, pelo entendimento de crescimento econômico como "processo", o que implica incorporar seu ambiente histórico e suas especificidades locais. Em segundo lugar, por conta destas especificidades, não se poderia pressupor que trajetórias individuais apresentadas por determinadas economias possam ser historicamente copiadas, revelando que o desdobramento institucional para o crescimento é necessariamente marcado pela "incerteza" e pela especificidade histórica. Em terceiro lugar, o processo de crescimento econômico, em que pese o amplo espectro de instituições - entendidas enquanto conjunto de normas, regras e hábitos e sua evolução (North, 1994, Hodgson, 1993) -, tem uma referência na ação individual dos agentes, que revela necessária e fundamentalmente a importância das firmas, organizações, e, portanto, do ambiente microeconômico na definição das diferentes trajetórias.

A questão da importância das instituições como fator de regulação do desempenho econômico merece alguns comentários. Como se verá na seqüência desse artigo, há entre as correntes institucionalistas uma permanente tensão entre duas grandes abordagens. De um lado, incluem-se os herdeiros da tradição mais próxima do Antigo Institucionalismo Norte-Americano, que tem nos estudos de Veblen, Commons e Mitchell, sua mais fiel representação. Hoje essa corrente, aceitando a proposição sugerida por Nelson e Sampat (2001, p. 18), incluiria autores como Geoffrey Hodgson, Thrainn Eggertsson, Malcolm Rutherford e

(4) Isso se manifesta apesar de se reconhecer que, quantitativamente, o crescimento econômico sempre se apresenta como um incremento na relação produto-capital, ou como aumento da acumulação de capital per capita superior ao crescimento populacional, ou ainda como crescimento da produtividade do capital em relação ao aumento da população.

(5) Tal subdivisão será mais bem explicada na seção seguinte. 
Richard Langlois. ${ }^{6}$ De outro lado, há um grupo de autores que, com exceção de Commons - o qual, segundo os mesmos, sugeriu a noção de custos de transação tem pouca afinidade com o Antigo Institucionalismo sendo, por isso mesmo, designados de teóricos da NEI. Incluir-se-iam nessa corrente autores como Demsetz, Alchian, Sudgen, Axelrod e outros. Todos aceitariam a proposição original de Coase - posteriormente desenvolvida por Williamson e North - que se centra na teoria dos custos de transação.

Para os seguidores do Antigo Institucionalismo de Veblen - como Hodgson, por exemplo -, há forte discordância em que os rígidos pressupostos da racionalidade (substantiva) da teoria econômica sejam capazes de proporcionar explicações factíveis e realísticas, no sentido de que o comportamento humano seja considerado efetivamente "eficaz", em contextos onde já exista uma considerável experiência comum. Já para os teóricos filiados à NEI, as instituições definem, modelam e mantêm o referido "comportamento racional" nos diferentes contextos: os indivíduos não deduzem ou pensam por si mesmos sobre o que é uma ação adequada, senão que atuam apenas fazendo o que é convencional no respectivo contexto (Nelson; Sampat, 2001).

Ainda que Commons aceite que os costumes, as normas e as regras possam surgir espontaneamente, assinala o decisivo papel da ação coletiva consciente do governo na solução de conflitos entre as instituições, o que sugere que o papel das instituições (e dos mecanismos de ação coletiva) é assegurar certa "ordem" ao funcionamento do sistema. As decisões daí derivadas, ao se converterem em lei ou apoiadas no plano da política, tornam, dessa forma, as referidas instituições mais específicas, precisas e duradouras.

Portanto, a diferença entre uma teoria que estabelece que as instituições implicam uma planificação consciente e coordenada e uma teoria que as concebe como resultado de um processo evolutivo não coordenado não se traduz necessariamente em uma diferença sobre se as instituições vigentes são "eficientes" ou não. Para a tradição institucionalista neoclássica, os trabalhos de Demsetz sobre direitos de propriedade incluíam a pressuposição de que "a lei era eficiente e que as mudanças legais refletiam mudanças em regras socialmente ótimas" (Nelson; Sampat, 2001, p. 240). Da mesma forma, parte dos estudos sobre organização dos negócios supõe que as formas organizacionais são escolhidas racionalmente, sendo, portanto, ótimas.

(6) A esta corrente contemporânea, que inclui grande parte dos institucionalistas críticos à visão defendida pelos expoentes da Nova Economia Institucional, temos designado, como foi sugerido no início desse artigo, de Neo-institucionalistas (Samuels, 1995). Tal denominação não é consensual nem entre os autores a ela vinculados, que preferem ser identificados como representantes do antigo institucionalismo de Veblen. Julga-se, entretanto, que vários conceitos e avanços teóricos alcançados por essa linha de pensamento permitem que se incluam seus expoentes em uma linha de pensamento diferente das proposições vigentes na época de Veblen. 
Atualmente, observa-se nas várias escolas institucionalistas, principalmente dentro da própria NEI, um afastamento dessas posições. Douglass North, que, nos primeiros estudos, supunha que as instituições evoluíam de forma a alcançar níveis mais elevados de eficiência (Davis; North, 1971 apud Nelson; Sampat, 2001, p. 25), tem defendido em seus estudos, como o de 1990, que sociedades que possuem instituições relativamente eficientes são relativamente mais afortunadas. Esse item será mais bem explorado em seu trabalho de 2005, conforme se analisará na seção seguinte. Nesse sentido, a idéia de que não é necessário que as instituições sejam eficientes desencadeia uma nova vertente teórica, segundo a qual as instituições vigentes são quem, em última instância, explica as diferenças de desempenho econômico entre os países, dentro dos quais as mesmas assumem distintos arranjos institucionais locais. É esse o caminho que começa a ser esboçado na análise de Matthews e que toma forma mais definida na análise de Zysman, conforme também o sugere Geoffrey Hodgson (1998). Os estudos mais recentes de North e Nelson revelam essa convergência. Depreende-se daí que "construir" um ambiente institucional adequado e mutante não implica necessariamente torná-lo mais eficiente: só a construção e evolução de mesmo poderá, no futuro, fornecer essa resposta, com base na experiência histórica adquirida.

\section{As abordagens institucionalistas e o processo de crescimento}

As abordagens institucionalistas têm contemplado em certa medida alguma teorização sobre o tema do crescimento econômico. Seus avanços, bem como seus limites, ocuparão este item. Nosso ponto de partida é o reconhecimento de que houve, nas últimas décadas, um revigoramento de estudos centrados nas instituições, consolidando seu campo de pesquisa (Hodgson, 1993; Samuels, 1995; Nelson, 1995; Matthews, 1986). Em função destes avanços algumas constatações podem ser feitas. Matthews (1986, p. 903) já reconhecia: “... the economics of institutions has become one of the liveliest areas of our discipline. (...) [And a] body of thinking has evolved based on two propositions: (i) institutions do matter, (ii) the determinants of institutions are susceptible to analysis by the tools of economic theory”. Portanto, há que se buscar interpretar que ferramentas são estas na compreensão do referido fenômeno.

No que tange às principais divergências, analisaremos e compararemos, como já foi dito, as abordagens institucionalistas de crescimento econômico sob três visões. A primeira é representada por Matthews, que tem uma filiação teórica próxima à NEI, mas que procura identificar as fontes de crescimento econômico, como algo distinto de qualquer semelhança com o ótimo paretiano. A segunda é a contribuição de Zysman (1994), de inclinação evolucionária, que se preocupa em desenvolver uma teorização sobre como as instituições criam "trajetórias de crescimento historicamente enraizadas”. Saliente-se que essa abordagem dá ênfase 
aos aspectos microeconômicos na definição das referidas trajetórias (global e/ou nacional). E a terceira é a análise de Douglass North, que se ocupa fundamentalmente com a noção de mudança institucional. As três abordagens não são excludentes, embora o que uma priorize a outra coloque em segundo plano. Mas elas concordam em alguns pontos, como a importância da mudança institucional e tecnológica como fator desencadeador do processo de crescimento. $\mathrm{O}$ que parece fundamental de se extrair dessa discussão é que crescimento enquanto processo exige a construção de um ambiente adequado, articulado macro e microeconomicamente, associado a uma noção de mudança institucional vinculado à abordagem evolucionária. $\mathrm{O}$ que se procurará constatar dessa discussão é que esses autores institucionalistas estão em linha de convergência com o campo de pesquisa evolucionário, que avança, conforme referido no estudo de Richard Nelson (2002), na construção de uma ponte entre a incorporação do conceito de instituição e a compreensão do processo de crescimento econômico.

\subsection{Matthews e as fontes do crescimento econômico}

Matthews (1986), embora reconheça certa convergência nas modernas abordagens institucionalistas, argumenta que há várias diferenças entre elas. A começar pelo próprio conceito de instituição, que, segundo ele, gravita em torno de três eixos. O primeiro identifica instituições econômicas alternativas como resultado de sistema de "direitos de propriedade" (property rights) alternativos. ${ }^{7} \mathrm{~A}$ segunda definição associa instituição a convenções ou normas de comportamento econômico, servindo como suporte à execução e cumprimento das leis. Nesta abordagem não há uma vinculação tão direta à economia dos custos de transação. $\mathrm{Na}$ França, desenvolveu-se uma derivação desta concepção constituindo a denominada "Economia das Convenções", cujo expoente é Olivier Favereau. E uma terceira derivação centra-se nos tipos de contrato, que pode se refletir em diferentes formas de autoridade. Estas são as razões que levam a definição de "instituição" a assumir conotações múltiplas. ${ }^{8}$

(7) Esta noção é particularmente importante para as abordagens seguidoras de Coase (1960), pois, para ele: “... any system of property rights is capable of leading to Pareto-efficiency provided it is a complete system, a complete system meaning one where all rights to all the benefits from all scarce resources are imputed to someone and are tradable; but that a complete system is never possible, because of transaction costs; and that some incomplete system, i.e. some institutions, are more conductive to Pareto-efficiency than others" (Matthews, 1986, p. 904).

(8) Completando essa definição Matthews (1986, p. 905) afirma que: "The word 'institution' is sometimes used in a quite different sense to mean an organisation. I shall not be using it in that sense, though a case can be made for regarding an organisation as consisting of a set of institutions in the sense I am using. The common feature of the four approaches I have enumerated - property rights, conventions, types of contract, and authority - is the concept of institutions as sets of rights and obligations affecting people in their economic lives. (...) A system of institutions can thus be described more or less equivalently in the legal kind of parlance I have been using, as the set of rights and obligations in force; or in the parlance of sociology and social anthropology, as a role-system or status-system; or in the parlance of economics, as defining: (i) what markets exist, (...) and (ii) how economic relations are regulated in areas where markets do not exist."

Economia e Sociedade, Campinas, v. 17, n. 1 (32), p. 85-105, abr. 2008. 
Sob essa perspectiva conceitual o fenômeno de crescimento econômico é entendido enquanto manifestação de mudanças institucionais. ${ }^{9}$ Vale dizer, o vínculo entre crescimento e instituições é realizado pelo conceito de mudança, que pressupõe inovações:

The analogy between institutional innovations and technical innovations creates a presumption, no more, that institutional change has made a positive contribution to economic growth. The presumption is that in the course of time people have discovered and adopted institutional arrangements that enabled them to co-operate with one another more efficiently than they did before (Matthews, 1986, p. 908).

Como a fonte do crescimento econômico é a mudança institucional, essa assume duas formas distintas. A idéia de ótimo paretiano pode estar presente, desde que mesclada com elementos evolucionários, como a noção de processo contínuo e múltiplos equilíbrios, o que a transforma radicalmente. Matthews conclui que o processo de mudança econômica, institucional e tecnológica é completamente diferenciado de um processo de melhoras sucessivas e adaptativas que levem a uma única situação de convergência ao ótimo paretiano. Isso porque ma realidade há uma série de fatores que obstaculizam tal perspectiva como o papel do Estado, interações não voluntárias, inércia e complexidade.

\subsection{Zysman: instituições e trajetórias históricas de crescimento}

A abordagem de John Zysman (1994) enfatiza que as trajetórias de crescimento são criadas historicamente, a partir do desenvolvimento de trajetórias nacionais institucionalmente inventadas ou enraizadas (Historically rooted trajectories of growth). Ou seja, as instituições importam, porque determinam diferentes trajetórias de crescimento econômico nos diversos ambientes nacionais. Há várias formas de se organizar as economias de mercado, os mercados são diferentes e há vários tipos de capitalismo. ${ }^{10}$

Esta abordagem procura associar mais diretamente institucionalismo à teoria econômica, estabelecendo nexos entre escolhas individuais, tipos de contrato e estrutura dos problemas enfrentados pelas suas respectivas empresas e organizações, originando as "instituições nacionais enraizadas historicamente". Pode-se tratar tal concepção como uma espécie de "institucionalismo histórico",

(9) O autor define crescimento econômico como tradicionalmente o faz o modelo neoclássico, ou seja, é uma medida (do crescimento) da renda per capita, onde se assume que "... Pareto-improvement conduces to economic growth so defined" (Matthews, 1986, p. 908).

(10) A abordagem institucional começa com a observação de que os mercados, incorporados (embedded) nas instituições políticas e sociais, são criações dos governantes e da política. Eles não existem ou operam fora das regras e instituições que os estabelecem e que estruturam o ato de comprar, vender e a própria organização da produção. Conseqüentemente, há múltiplos capitalismos de mercado, e em uma economia internacional global a competição entre seus membros deve ser entendida como uma interação entre os vários sistemas nacionais de mercado (Zysman, 1994, p. 243). Vide também Hodgson (1996). 
sem deixar de referir que levanta problemas e propõe soluções considerando aspectos relacionados ao microeconomic-based institutionalism. Neste sentido, diferentes conformações históricas e institucionais desenham, nos diversos contextos regionais, os sistemas nacionais de inovação, que distinguem as trajetórias tecnológicas. Por isto, institucionalismo e evolucionismo são fenômenos impossíveis de serem compreendidos de maneira desvinculada.

O ponto de partida da Historically rooted trajectories of growth é a recorrente crítica à noção de crescimento nas novas teorias de crescimento endógeno. Para Zysman, tanto o argumento de Romer, quanto o das informações assimétricas de Stiglitz estão assentados em pressupostos de equilíbrio. Ao contrário, o pensamento evolucionário inspira-se nas particulares e, portanto, múltiplas, trajetórias nacionais, que independem do equilíbrio de steady state. Por esta razão, o reconhecimento implícito de trajetórias de crescimento abre espaço para que as instituições façam a mediação entre estas trajetórias, permitindo que se delineiem formas diferenciadas de desenvolvimento econômico.

Neste contexto, é importante mencionar que são as estratégias, quer em nível empresarial, quer governamental, que, ao exercerem influência decisiva sobre as inovações, formam um ambiente adequado para os novos produtos e processos. Estabelece-se daí um importante ponto da passagem micro para a macro, pois não é o governo quem define estratégias para as firmas implementarem, mas o contrário, pois analiticamente o salto se manifesta do particular para o geral. Em outros termos, a capacidade do governo em produzir resultados em mercados específicos não cria inevitavelmente vantagens de crescimento no mais longo prazo, e, alternativamente, seu fracasso em gerar ou criar vantagens não produz inevitavelmente desvantagens.

$\mathrm{O}$ argumento levantado permite concluir que as histórias ou movimentos nacionais são parte de um processo de interação e competição. Daí se estabelece que: a) diferentes 'lógicas de mercado' têm efeitos de longo-prazo no tipo, padrão ou modelo, e nas taxas de crescimento em cada economia; b) o caráter de interação da lógica nacional de mercado entre um país e seus principais parceiros comerciais pode influenciar o caráter do crescimento de cada economia; e c) a lógica de mercado das economias dominantes nacionais podem influenciar a economia mundial como um todo (Zysman, 1994, p. 255).

Tais conclusões requalificam o debate sobre formas alternativas de crescimento, colocando o mercado e suas especificidades nacionais como fator condicionante primordial a tal objetivo. Entretanto, tal entidade (ou, melhor dizendo, instituição), deve ser entendida não como um princípio regulador e racionalizador de decisões ótimas, mas como produto de interações, estratégias, decisões frente à incerteza que repercutem, favoravelmente ou não, através da 
atuação de toda uma rede institucional, que lhe assegura sustentabilidade. Por esta razão, a noção de mercado é indissociável da noção de instituição, pois a primeira, mais do que produto da segunda, é sua própria manifestação.

Segundo o modelo proposto por Zysman, as trajetórias de crescimento cujas instituições são fontes geradoras - se dão tanto pela existência de padrões de inovação, quanto pelo desenvolvimento tecnológico. Através de rotinas e políticas específicas estabelecem-se os termos do desenvolvimento econômico. ${ }^{11}$ A opção que determina quem é perdedor ou ganhador torna-se parte do problema de alocação de custos nas mudanças industriais, envolvendo, independente do modelo de desenvolvimento industrial adotado, três aspectos sempre presentes: capacidade técnica da ação do Estado na economia; estabelecimento de uma política de alocação de custos da mudança industrial; e processo político para permitir tais cumprimentos.

As estruturas institucionais nacionais são resultantes do processo histórico de desenvolvimento industrial e da modernização política, o que está diretamente associado à argumentação evolucionária das "trajetórias tecnológicas", que levam em conta fatores essenciais como o processo de difusão da informação e o de geração de novas idéias.

Esta é a idéia motora da abordagem de crescimento institucional, pois não basta a geração de investimento para criar as bases para um processo de crescimento. Faz-se necessária a construção de um ambiente institucional adequado capaz de transformá-lo em crescimento, o que obviamente implica em uma série de outros fatores:

Technology, like market processes, is not disembodied. It develops in communities; it has local roots. The processes of learning that drive its development are shaped by the community and institutional structure, and consequently the technological trajectories can only be defined in reference to particular societies (Zysman, 1994, p. 261).

Portanto, as instituições não são neutras e podem proporcionar explicações sobre trajetórias específicas. Assim, uma dada estrutura política e institucional induz à formação de uma lógica de mercado que orienta e dirige a trajetória de crescimento. Tal constatação, mesmo que ainda limitada (porque não explica como o contexto nacional afeta as estratégias das firmas, ou se as explica o faz em

(11) Para Zysman (1994, p. 257), o processo de desenvolvimento é inerentemente desordenado e doloroso, onde os trabalhadores ou administradores especializados são desvalorizados com a perda de seus empregos e renda. O problema político é resolver quem ganha e perde com o crescimento. Conflitos intermináveis em torno dos ganhos e dores do crescimento podem interferir no crescimento através da ruptura do próprio processo de ajustamento de mercado. Greves na indústria, protestos dos proprietários rurais, lobbies em favor de regras que preservem posições no mercado ou que favoreçam às novas industrias são expressões das políticas de ajustamento, esforços que visam determinar quem ganha e perde com o crescimento. 
um contexto demasiadamente amplo que inclui tudo na referida lista), revela certo avanço analítico.

Pelo exposto, depreende-se que o ideário institucionalista está mais próximo do campo analítico heterodoxo do que no mainstream neoclássico, visto que seus princípios teóricos originaram-se da oposição aos fundamentos de equilíbrio, otimalidade e racionalidade substantiva. Qualquer abordagem analítica que se pretenda institucionalista deve incluir path dependency, reconhecer o caráter irreversivelmente diferenciado do processo de desenvolvimento econômico e pressupor que o ambiente econômico envolve disputas, antagonismos, conflitos e incerteza. Nesse sentido, a adversidade, a adaptação e a seleção são elementos fundamentais à definição de estratégias empresariais e às trajetórias de crescimento econômico.

\subsection{North e o papel da mudança institucional}

Para Douglass North (1990), o fundamental no campo do desenvolvimento econômico é buscar a formulação de uma ainda inexistente "teoria da dinâmica econômica". E esta reside fundamentalmente na compreensão e sistematização do processo de mudança. ${ }^{12}$ Em sendo assim, as trajetórias das mudanças institucionais são elementos essenciais na definição das diferentes formas de crescimento econômico, o que revela notável semelhança com o pensamento evolucionário:

If we look back far enough in history, divergence appears to be very simple to explain. (...) However, after ten thousand years of civilization, despite the immense decline in information costs and despite the implications of neoclassical international trade models that would suggest convergence, there is enormous contrast between economics (North, 1990, p. 92).

Para North, a mudança econômica de longo prazo é uma "conseqüência cumulativa" de inúmeras decisões de curto prazo tomadas por políticos e empresários, que, direta ou indiretamente (via efeitos externos), determinam a performance econômica. Entretanto, o grau, através do qual os resultados são consistentes com as intenções, refletirá o grau através do qual os modelos dos empresários são efetivamente "verdadeiros". Isto porque os modelos refletem idéias, ideologias e crenças que são, na melhor das hipóteses, apenas parcialmente refinadas e melhoradas por feedback de informações sobre as consequiências atuais das políticas tornadas legitimamente legais. Em outros termos, as conseqüências

(12) Em suas palavras: "A theory of economic dynamics is also crucial for the field of economic development. There is no mystery why the field of development has failed to develop during the five decades since the end of World War II. Neoclassical theory is simply an inappropriate tool to analyze and prescribe policies that will induce development. It is concerned with the operation of markets, not with how markets develop. How can one prescribe policies when one doesn't understand how economies develop?" (North, 1994, p. 359). 
de políticas específicas não são apenas incertas, mas imprevisíveis. Por essa razão, conclui North que:

Even the most casual inspection of political and economic choices, both throughout history and today, makes clear the wide gap between intentions and outcomes. However, the increasing-returns characteristics of the institutional matrix and the complementary subjective models of the players suggest that although the specific short-run paths are unforeseeable, the overall direction in the long run is both more predictable and more difficult to reverse (North, 1990, p. 104).

Em seu livro de 2005, North reforça a argumentação da necessidade em se compreender o processo de mudança econômica como principal fonte de explicação dos fenômenos vinculados ao processo de crescimento. ${ }^{13}$ Ao tentar desvendar a lógica de tão complexo processo, que necessariamente deve contemplar analiticamente aspectos institucionais relevantes e de difícil sistematização, North, mais uma vez, confronta tal necessidade com a fragilidade do instrumental neoclássico, apesar de seus notáveis avanços na área quantitativa. $\mathrm{Na}$ estrutura do referido livro, salienta que o processo de mudança econômica (e institucional) deve, necessariamente, contemplar os seguintes aspectos: a incerteza em um mundo não ergódico; os sistemas de crenças, cultura e ciência cognitiva; a consciência e a intencionalidade humanas. Esses aspectos, em conjunto, definem o que ele designa de arcabouço de interações humanas que permitem a construção da estrutura institucional.

No respectivo capítulo, denominado The scaffolds humans erect, observa que a mudança institucional segue cinco proposições, que se centram na importância da competitividade, do conhecimento, da estrutura de incentivos e das formas de percepção dos agentes. As proposições de North são:

1. The continuous interaction between institutions and organizations in the economic setting of scarcity and hence competition is the key to institutional change. 2. Competition forces organizations to continually invest in skills and knowledge to survive. The kinds of skills and knowledge individuals and their organizations acquire will shape evolving perceptions about opportunities and hence choices that will incrementally alter institutions. 3. The institutional framework provides the incentives that dictate the kinds of skills and knowledge perceived to have the maximum pay-off. 4. Perceptions are derived from the mental constructs of the players. 5. The economies of scope, complementarities, and network externalities of an institutional matrix make institutional change overwhelmingly incremental and path dependent (North, 2005, p. 59).

(13) Segundo ele: "Understanding the process of economic change would enable us to account for the diverse performance of economies, past and present. We would be able to account for the long history of sustained growth of the United States and western Europe, the spectacular rise and demise of the Soviet Union, for the contrasting performances of rapid economic growth of Taiwan and South Korea and the dismal record of sub-Saharan Africa economies, and the contrasting evolution of Latin America and of North America" (North, 2005, p. vii). 
Após discorrer sobre cada um desses itens, North salienta que: "[t]his characterization of institutional change is a major building block in our construction of an understanding of the process of economic change" (North, 2005, p. 64). Essa afirmativa revela importante insight a respeito da complexa relação instituição e crescimento econômico, que tem na mudança institucional seu traço mais revelador.

\section{A contribuição evolucionária de Nelson}

Nelson (2002) argumenta que os economistas que vêm mais contribuindo para o desenvolvimento da teoria de crescimento evolucionária, ao longo dos últimos vinte anos, têm se motivado nessa tarefa pela percepção de que a teoria neoclássica de crescimento econômico, embora assinalando que a mudança tecnológica exerce papel central no crescimento econômico, é totalmente inadequada ao caracterizar abstratamente tal processo. Mais especificamente, tal teoria é incapaz de caracterizar o processo de crescimento econômico desencadeado pela mudança tecnológica, como ele próprio o faz em conjunto com Winter (Nelson e Winter, 1982). Em particular, o modelo neoclássico desconsidera o fato de que os esforços para o avanço tecnológico são, em grande medida, "cegos" (Nelson, 2002, p. 17).

Tal proposição não implica negar o propósito, a inteligência e o corpo de entendimento das tentativas em se avançar na forma de incorporar o avanço tecnológico. É o caso de diferentes inventores e equipes de $\mathrm{P} \& \mathrm{D}$, que permitiram avanços em diferentes áreas, cujas conquistas são virtualmente impossíveis de serem previstas, no sentido de captar o avanço inerente a elas. A noção de que o avanço tecnológico se dá através de um processo evolucionário tem se desenvolvido de forma independente por estudiosos que atuam em uma variedade de disciplinas, como na sociologia (Constant, 1980; Bijker, 1995), nos historiadores da tecnologia (Rosenberg, 1976; Vincenti, 1990; Petrosli, 1992; Mokyr, 1990), bem como por economistas interessados em modelagem (Nelson e Winter, 1982; Metcalfe, 1998; Saviotti, 1996).

Isto implica reconhecer que o procedimento do avanço tecnológico em um processo evolucionário leva à formulação de uma teoria do crescimento que tem uma estrutura muito diferente da existente na teoria de crescimento neoclássica, tanto a velha, como a nova. Contudo, como salienta Nelson (2002, p. 18): "However, for the most part evolutionary growth theory, like neoclassical growth theory, has not as yet taken on board the complex institutional structures that are characteristic of modern economies".

Por outro lado, sofisticados estudos empíricos sobre os avanços tecnológicos compreendem que o padrão e a característica de tal processo foi 
influenciado pela estrutura institucional que os sustenta e que as instituições também condicionam fortemente a forma como as novas tecnologias são aceitas e absorvidas pelo sistema econômico. Esse ponto é claramente evidenciado em estudos como os de David Landes (Unbounded prometheus, 1970), de Christopher Freeman (The economics of industrial innovation, 1982) e mais recentemente, pelos conceitos de sistema nacional (ou setorial) de inovação (Lundvall, 1992; Nelson, 1993; Mowery; Nelson, 1999). ${ }^{14}$ Pode-se incluir, também, nessa lista, a relevante contribuição de neo-schumpeterianos ou evolucionários, que tem avançado na teorização de processos de aprendizado e capacitação tecnológica, como é o caso dos estudos de Dosi (1988) e Silverberg; Dosi e Orsenigo (1988).

Entretanto, deve se reconhecer que a contribuição dos modernos economistas evolucionários sobre mudança tecnológica, preocupados com o papel das instituições no desenvolvimento econômico, tem tido poucos interlocutores. Por essa razão, Nelson (2002, p. 18) considera que a contribuição de seu artigo é servir como uma espécie de ponte entre as duas referidas tradições intelectuais e sugerir um caminho em que ambas possam seguir conjuntamente. Tal conjugação, para as tradições evolucionárias e institucionalistas, não constitui novidade porque, mesmo antes que a moderna teoria neoclássica ganhasse a atual preponderância, a maior parte da análise econômica era tanto evolucionária, quanto institucionalista. Por mais estranho que essa afirmação possa parecer aos céticos em relação a essa abordagem:

Thus, Adam Smith's analysis concerned with how 'the division of labor is limited by the extent of the market' and, in particular, his famous pinmaking example, certainly fits the mold of what I would call evolutionary theorizing about economic change. Indeed, his analysis is very much one about the co-evolution of physical technologies and the organization of work, with the latter, I would argue, very much a notion about 'institutions'. In many other places in 'The Wealth of Nations', Smith is expressly concerned with the broader institutional structure of nations, in a way that certainly is consonant with the perspectives of modern institutional economics. Karl Marx of course was both an evolutionary theorist and an institutional theorist. If you consider the broad scan of his writing, so too was Alfred Marshall. Thus, evolutionary growth theorizing that encompasses institutions in an essential way has a long and honorable tradition in economics (Nelson, 2002, p. 18-19).

Sob esse aspecto, a ascensão hegemônica da teoria econômica neoclássica reduziu notavelmente o escopo intelectual implícito nas referidas abordagens, tornando a análise econômica evolucionária e institucionalista um corpo de estranho à teoria econômica. A tendência de dissonância em relação a essa hegemonia, no sentido de consolidação de uma trajetória própria, começou a

(14) Segundo Nelson (2002, p. 18): “(...) the notion of a national or a sectoral innovation system, which clearly is an institutional concept, has a played a significant role in theorizing about technological advance (see e.g. Lundvall, 1992; Nelson, 1993; Mowery and Nelson, 1999)". 
ganhar espaço nos Estados Unidos principalmente com a contribuição de Commons, que ajudou a definir a escola institucional Americana. Sua análise, entretanto, segundo Nelson, não era muito evolucionária. Nem Coase, que mais tarde deu substância à Nova Economia Institucional, assumiu tal caráter. ${ }^{15}$

Tanto a chamada Nova Economia Institucional, quanto a "Nova Economia Evolucionária" têm diferentes fontes e enfoques: a primeira ocupa-se com o conjunto de fatores que moldam e definem as interações humanas (segundo Commons) tanto dentro das organizações, como entre elas; e a última ocupa-se fundamentalmente com o processo de avanço tecnológico. Entretanto, mais recentemente, o desenvolvimento em ambos os campos vêm revelando ampla convergência, como os trabalhos de Hodgson (1988, 1993) e Langlois (1989) o revelam. Também os trabalhos de Douglass North, como se viu no item anterior, vem hoje apresentando grande proximidade teórica com a perspectiva evolucionária, notadamente ao enfatizar a maneira pela qual as instituições se formam e se modificam. Para Nelson:

Thus, Douglass North (1990), perhaps today's best known economic 'institutionalist', gradually has adopted an evolutionary perspective regarding how institutions form and change. And, as I noted earlier, many of the scholars who did the early work on the new evolutionary economics recently have become focused on such subjects as the 'national innovation systems', which is an institutional concept par excellence (Nelson, 2002, p. 19).

Há fortes afinidades entre a forma comum do core de pressupostos e percepções entre economia institucional - pelo menos aqueles pertencentes à escola de North - e a moderna economia evolucionária. Por isso há fortes razões para que ambas correntes juntem suas forças: ambos têm uma premissa central de que a ação humana e as interações são resultantes e resultam de hábitos de ação e de pensamento. Isto implica a rejeição do princípio de "maximização" enquanto "processo" de caracterização da forma como os homens agem. Há também a rejeição da noção friedmaniana, segundo a qual, se os homens não se orientam através do cálculo maximizador, comportam-se "como se" - o princípio do as if o fizessem. Para os campos de pesquisa institucionalistas, quanto evolucionários:

...patterns of action need to be understood in behavioral terms, with improvements over time being explained as occurring through process of individual and collective learning. For economic evolutionary theorists, this exactly defines the nature of an evolutionary process (Nelson, 2002, p. 20).

(15) Surpreendentemente e apesar de Schumpeter em seus trabalhos criticar o Antigo Institucionalismo por falta de uma teoria que lhe permitisse avançar teoricamente (Hodgson, 1998), Nelson enfatiza que: "Schumpeter (1942), whose work arguably has provided the starting point for modern evolutionary economics, is seldom footnoted by self-professed institutionalists, despite the fact that Schumpeter was very much concerned with economic institutions. And Schumpeter's institutional orientation was ignored, as well, in the early writings of the evolutionary economists who cited Schumpeter as their inspiration" (Nelson, 2002, p. 19).

Economia e Sociedade, Campinas, v. 17, n. 1 (32), p. 85-105, abr. 2008. 
Por essas razões, os estudiosos desses dois campos têm aumentado seus interesses na compreensão dos determinantes da performance econômica, e como essa diferencia as nações ao longo do tempo. Os modernos teóricos evolucionários enfocam centralmente o que chamam de "tecnologias". ${ }^{16} \mathrm{E}$ os economistas institucionalistas enfocam predominantemente as instituições. ${ }^{17}$ Para Nelson, o casamento entre ambos é bastante viável: "Below I map out what a marriage might look like" (Nelson, 2002, p. 20). Para tanto, faz-se necessária a incorporação de alguns conceitos, como o das "rotinas", enquanto conceito unificador. Depois, propõe o conceito de "tecnologia social", como inerentemente vinculado às instituições. E, finalmente, incorpora as instituições em uma teoria evolucionária de crescimento econômico.

\subsection{Por que as rotinas importam?}

O conceito de rotina foi proposto por Nelson e Winter (1982) da seguinte forma: “... the carrying out of a routine is 'programmatic' in nature, and like a program tends largely to be carried out automatically. Like a computer program, our routine concept admits choice within a limited range of alternatives, but channeled choice" (Nelson, 2002, p. 20).

Tal conceito foi construído a partir da firma de negócios, empresa ou outra organização que desenvolva atividade econômica e determina a forma como ela age, sob circunstâncias particulares com as quais a mesma se defronta. Sua performance será determinada pelas rotinas que possui e pelas rotinas possuídas por outras firmas com as quais interage, incluindo competidores, fornecedores e clientes. Em qualquer período dado de tempo, muitas das rotinas são largamente comuns às firmas da mesma área, mas algumas vezes não: nesses casos, são as mesmas rotinas que irão fornecer a "matéria-prima" (stuff), que determinará a forma como as firmas agirão em relação aos seus competidores. A distribuição de rotinas em uma economia, em qualquer momento, determina a performance econômica global. ${ }^{18}$ Observe-se a importância conferida às organizações para a

(16) No original, Nelson (2002, p. 20) afirma: "For evolutionary theorists, a country's level of technological competence is seen as the basic factor constraining it's, with technological advance the central driving force behind economic growth. As noted, increasingly evolutionary economists are coming to see "institutions" as molding the technologies used by a society, and technological change itself. However, institutions have not as yet been incorporated into their formal analysis."

(17) No original, Nelson (2002, p. 20) afirma: "Many would be happy to admit that the influence of a countries institutions on it's ability to master and advance technology is a central way that institutions affect economic performance. However, institutionalists have yet to include technology and technological change explicitly into their formulation."

(18) Em suas palavras: "Under evolutionary economic theory, economic growth is caused by changes in the distribution of operative routines, associated both with the creation of superior new routines, and the increasingly widespread use of superior routines and the abandonment of inferior ones. The latter can occur through the relative expansion of organizations that do well, or the adoption of better techniques by organizations that had been using less good ones, or both" (Nelson, 2002, p. 20). 
performance econômica do ponto de vista mais global, o que explicita a relevância dos aspectos microeconômicos em relação ao plano macro. Mais ainda, Nelson reconhece que o conceito de rotina aproxima mais a economia evolucionária dos institucionalistas, pois:

As noted, most of the writing by evolutionary economists has focused on 'physical' technologies as routines. However, the notion of a routine fits very well with the conceptualization of many institutional economists, if the concept is turned to characterize standardized patterns of human transaction and interaction more generally. Indeed, if one defines institutions as widely employed 'social' technologies, in the sense I will develop shortly, it is easy to take institutions on board as a component of an evolutionary theory of economic growth (Nelson, 2002, p. 21).

Dentre as principais características das "rotinas produtivas" tem-se: uma coleção de procedimentos, que, tomados em conjunto, resultam em um prognosticável e especificável resultado; rotinas complexas quase sempre podem ser analiticamente desdobráveis em uma série de sub-rotinas (por exemplo, para se fazer um bolo leva-se em conta sub-rotinas como preparar a massa, misturá-la e cozinhá-la; essas, por sua vez, envolvem farinha, açúcar e fogão); logo, virtualmente, todas as rotinas complexas estão vinculadas a outras que devem ser efetivadas de forma a torná-las possíveis, ou incapacitando-as a criar valor.

Um outro aspecto-chave das rotinas produtivas é que, enquanto a operação de uma rotina particular por um concorrente individual ou organização, geralmente, envolve certos elementos idiossincráticos, o seu núcleo, normalmente, inclui elementos que são bastante similares uns aos outros, e que operam em um mesmo contexto. Como exemplo, cita que os ingredientes e os equipamentos usados por padeiros razoavelmente especializados são basicamente os mesmos usados por outros confeiteiros especializados.

Há duas razões para que as rotinas produtivas sejam disseminadas por aqueles que são especializados na referida "arte": a primeira resulta das contribuições cumulativas das muitas partes, freqüentemente operando por várias gerações (desviar das mesmas significa alto risco, e mesmo que os payoffs possam ser consideráveis, há maior chance de fracasso); a segunda é que uma rotina particular tende a fazer parte de um sistema de rotinas. Esse aspecto sistêmico força uma certa generalidade de formas de se fazer coisas particulares.

\subsection{Tecnologia social e instituições}

O conceito de tecnologia social é proposto em Nelson e Sampat (2001), e construído sob o pressuposto de que as rotinas envolvem dois aspectos: uma receita que é anônima com referência à qualquer divisão do trabalho e uma divisão do trabalho plus um modo de coordenação. No primeiro caso, inclui-se o que os 
estudiosos freqüentemente têm em mente quando se referem às "tecnologias físicas". E, no segundo, tem-se as "tecnologias sociais", que se referem ao que os estudiosos têm em mente quando se referem às instituições. Segundo Nelson (2002, p. 22), North e Wallis (1994) propuseram uma distinção semelhante. Em sentido amplo, as tecnologias sociais podem definir e serem definidas como as regras do jogo, ou, numa alusão à proposição de Williamson, como "modo de governança". Em termos de custos de transação, tal conceito proporciona formas de se obter baixos custos de transação. Tal conceito é amplo suficiente para incorporar formas de organização da atividade econômica dentro de organizações particulares, como a forma M. Desta maneira, os mercados definem e são definidos pelas "tecnologias sociais", incorporando procedimentos utilizados pela escolha e ação coletiva.

Esta formulação naturalmente induz a que se percebam as instituições não tanto quanto "restrições" de comportamento, mas como formas alternativas de se obter coisas quando a cooperação humana é necessária. ${ }^{19}$ Essa importante conclusão remete para a concepção das tecnologias e instituições como necessariamente produtos da ação coletiva e interação social.

\subsection{Instituições em uma teoria econômica de crescimento}

A questão de como as instituições se ajustam a uma teoria de crescimento econômico depende não somente da forma como a definimos, mas de outros aspectos teóricos, como entender a forma como a concepção de instituição, enquanto "tecnologias sociais", se adapta às teorias evolucionárias de crescimento econômico. Nesse sentido, o avanço tecnológico retoma sua importância fundamental, com peso especial dentro da abordagem evolucionária:

(...) the reason is that, while neo-classical theory sees economic actors as facing a spacious choice set, including possible actions that they never have taken before, within they can choose with confidence and competence, evolutionary theory sees economic actors as at any time bound by the limited range of routines they have mastered. Each of these has only a small range of choice. Further, the learning of new routines by actors is a time consuming, costly, and risky thing. Thus while neoclassical growth theory sees considerable economic growth as possible simply by moving along the production function, in evolutionary theory there are no easy ways to come to master new things (Nelson, 2002, p. 23).

Em outros termos, sob a perspectiva evolucionária, o crescimento econômico precisa ser compreendido como resultado da progressiva introdução de

(19) Nesse sentido, textualmente, Nelson (2002, p. 22) afirma que: “(...) to view institutions as "constraints" on behavior is analogous to seeing prevailing physical technologies as constraints. A productive social technology (an institution) or a physical technology is like a paved road across a swamp. To say that the location of the prevailing road is a constraint on getting across is basically to miss the point. Without a road, getting across would be impossible, or at least much harder". 
novas tecnologias, associadas a níveis crescentemente mais elevados da produtividade do trabalho, à habilidade em se produzir novos e melhores bens e serviços, que são progressivamente capital-intensivo. Nessa formulação, as novas "instituições" e tecnologias sociais aparecem como mudanças nos modos de interação - novos modos de organização do trabalho, novos tipos de mercados, novas leis, novas formas de ação coletiva - que são chamadas, como as novas tecnologias, a trazer novos usos econômicos: "In turn, the institutional structure at any time has a profound effect on, and reflects, the technologies that are in use, and which are being developed" (Nelson, 2002, p. 23). Portanto, o conceito de instituições enquanto "tecnologias sociais", a linguagem das rotinas para descrevêlas e a teoria esquematizada acima sobre como as instituições e a mudança institucional emergem com o avanço das tecnologias físicas no processo de crescimento econômico, vêm se tornando noções bastante poderosas, aproximando a análise da forma de ação das referidas "tecnologias sociais".

\section{Considerações finais}

A generalidade e amplitude das proposições sobre arranjos institucionais para o crescimento econômico reforçam a necessidade em se explorar melhor os vínculos teóricos daí decorrentes, os quais necessariamente se materializarão em receitas e proposições específicas. Explicando melhor: os receituários das políticas econômicas adotadas nas várias economias mundiais incluem invariavelmente "desenhos institucionais" que levam em conta aspectos mais ou menos próximos de uma das (ou das três) correntes institucionalistas mencionadas no item 2 - quais sejam o Antigo Institucionalismo, a Nova Economia Institucional e o NeoInstitucionalismo. $\mathrm{O}$ modismo decorrente do sucesso que a NEI vem fazendo, principalmente a partir da concessão do Prêmio Nobel de Economia a Ronald Coase em 1991, e a Douglass North em 1993, levou suas análises a assumir um caráter cada vez mais propositivo, em termos dos fundamentos institucionais para erguer as condições de crescimento econômico e estabilidade nas várias economias. O mesmo ocorre, porém de forma mais tímida, com as contribuições neo-institucionalistas, que, grosso modo, advogam propostas teóricas que contemplem os avanços tecnológicos, a inovatividade, o catching up, as políticas distributivas keynesianas e instituições vinculadas (embedded) social e historicamente em suas respectivas realidades regionais.

Em geral, é de esperar que correntes mais heterodoxas pendam mais para o Antigo Institucionalismo de Veblen, e, conseqüentemente, para as modernas abordagens próximas ao neo-institucionalismo, ao passo que as mais ortodoxas penderão mais para uma "visão institucional" mais próxima à NEI.

Entretanto, os avanços teóricos sobre instituições e crescimento econômico não podem pretender se originar de uma única abordagem. Tal opção 
está longe de ser exeqüível, se for compreendida através da adoção de uma postura de excludência analítica. Julga-se que, pelo contrário, o processo de compreensão da mudança e do crescimento econômico deve ser construído através do exercício permanente da crítica e da reflexão, através da proposição contínua de novos conceitos e teorizações. Alguns avanços nesse sentido parecem definitivos. Por exemplo, Matthews enfatizou que o ambiente institucional, em que pese a importância das mudanças, não se aproxima, nem tende a se reproduzir, de maneira compatível com um ótimo paretiano. Zysman explicitou o nexo entre mudança institucional, crescimento econômico e o caráter histórico dessa determinação, como decorrente da supremacia microeconômica, em um ambiente evolucionário, sobre os demais aspectos, o que asseguraria a dinâmica da referido processo. E, para North, as instituições importam porque a mudança institucional assume um papel mais decisivo que as mudanças tecnológicas. Salientou também que a performance econômica dos países está diretamente associada ao padrão de mudanças institucionais regionalmente localizado. Nelson, nesse sentido, vem percebendo a constituição de uma nova agenda para a elaboração de uma teoria alternativa de crescimento econômico, que contemple, necessariamente, a abertura para os aspectos institucionais mencionados, junto com a noção de rotinas e do que ele designa como "tecnologia social". O reconhecimento da importância dessas observações deveria ser incorporado em qualquer análise centralizada na proposição de diferentes arranjos institucionais.

\section{Referências bibliográficas}

DOSI, Giovanni. Sources, procedures, and microeconomic effects of innovation. Journal of Economic Literature, v. 26, n. 3, p. 1120-1171, Sept., 1988.

HODGSON, Geoffrey M. Institutional economics: surveying the 'old' and the 'new'. Metroeconomica, v. 44, n. 1, p. 1-28, 1993.

Varieties of capitalism and varieties of economic theory. Review of International Political Economy, v. 3, n. 3, p. 380-433, Autumn 1996.

The approach of institutional economics. Journal of Economic Literature, v. 36, p. 166-192, Mar. 1998.

How economics forgot history: the problem of historical specificity in social science. London: Routledge, 2001.

MATTHEWS, R. C. O. The economics of institutions and the sources of growth. The Economic Journal, v. 96, p. 903-918, Dec. 1986.

NELSON, Richard R. Evolutionary theorizing about economic change. In: SMELSER, Neil J.; SWEDBERG, Richard (Ed.). The handbook of economic sociology. New York: Princeton University Press, Princeton \& Russel Sage Foundation, 1994. p. 108-136.

Recent evolutionary theorizing about economic change. Journal of Economic

Literature, v. 33, p. 48-90, Mar. 1995. 
NELSON, Richard R. The agenda for growth theory: a different point of view. Cambridge Journal of Economics, v. 22, p. 497-520, 1998.

Bringing institutions into evolutionary growth theory. Journal of Evolutionary Economics, Spring-Verlag, v. 12, p. 17-28, 2002.

; SAMPAT, Bhaven. Las instituciones como factor que regula el desempeño económico. Revista de Economía Institucional, n. 5, Segundo Semestre, 2001.

; WINTER, Sidney G. Evolutionary theorizing in economics. Journal of Economic Perspectives. v. 16, n. 2, p. 23-46, Spring 2002.

NORTH, Douglass C. Institutions, institutional change and economic performance. New York: Cambridge University Press, 1990.

. Understanding the process of economic change. Princeton and Oxford: Princeton University Press, 2005.

SILVERBERG, G.; DOSI, G.; ORSENIGO, L. Innovation, diversity and diffusion: a selforganization model. The Economic Journal, v. 98, n. 393, p. 1032-1054. Dec. 1988.

SAMUELS, Warren J. The present state of institutional economics. Cambridge Journal of Economics, v. 19, p. 569-590, 1995.

WILLIAMSON, Oliver E. Las instituciones económicas del capitalismo. México: Fondo de Cultura Económica, 1985.

Transaction cost economics and organization theory. Industrial and Corporate Change, v. 2, n. 2, p. 107-156, 1993.

Transaction cost economics. In: SMELSER, Neil J.; SWEDBERG, Richard (Ed.). The handbook of economic sociology. New York: Princeton University Press, Princeton \& Russel Sage Foundation, 1994. p. 77-107.

Hierarquies, markets and power in the economy: an economic perspective. Industrial and Corporate Change, v. 4, n. 1, p. 21-49, 1995.

ZYSMAN, John. How institutions create historically rooted trajectories of growth. Industrial and Corporate Change, v. 3, n. 1, p. 243-283, 1994. 